\title{
Task-specific elevation of motor learning by conjunctive transcranial direct current stimulation
}

\author{
Short title: Conjunctive tDCS enhanced motor learning
}

\author{
Ying Wang ${ }^{1,2,3^{*}}$, Ji-xian Wang ${ }^{4 *}$, Qing-fang Zhang ${ }^{1}$, Ke-wei Xiao ${ }^{1}$, Liang Wang ${ }^{1}$, \\ Qing-ping Yü ${ }^{1}$ Qing Xie ${ }^{4}$, Mu-ming Poo ${ }^{1,2,3,5 \#}$ and Yunqing Wen ${ }^{1 \#}$ \\ ${ }^{1}$ Institute of Neuroscience, State Key Laboratory of Neuroscience, CAS center for Excellence \\ in Brain Science \& Intelligence Technology, Chinese Academy of Sciences, Shanghai 200031, \\ China. \\ ${ }^{2}$ University of Chinese Academy of Sciences, Beijing 100049, China. \\ ${ }^{3}$ School of Life Science and Technology, ShanghaiTech University, Shanghai 201210, China. \\ ${ }^{4}$ Department of Rehabilitation Medicine, Ruijin Hospital, Shanghai Jiao Tong University \\ School of Medicine, Shanghai 200025, China. \\ ${ }^{5}$ Shanghai Center for Brain Science and Brain-Inspired Technology, Shanghai 200031, China.
}

* These authors contributed equally to this work.

\# Send communication to Y. Wen (wenyq@ion.ac.cn) and M-m.Poo (mpoo@ion.ac.cn) 
Transcranial direct current stimulation (tDCS) is used for modulating brain functions, but the optimal protocol remains unclear. We found that mouse learning to run on a rotarod was enhanced by anodal tDCS during ("online") but not before or after ("offline") the task performance. The enhancement was task-specific, since online tDCS during rotarod learning did not affect learning of beam walking, and vice versa. For mice underwent middle cerebral artery occlusion (MCAO), online anodal tDCS restored motor learning capability in a task-specific manner. Transcranial calcium imaging showed that anodal and cathodal tDCS elevated and suppressed cortical neuronal activity, respectively, suggesting that elevated spiking in task-activated neural circuits underlie the learning enhancement. Thus, the efficacy of tDCS could be elevated by conjunctive activation of targeted neural circuits.

One sentence summary: Transcranial direct current stimulation applied in temporal conjunction with the motor task enhances motor learning in a task-specific manner.

Transcranial direct current stimulation (tDCS) is now widely used for non-invasive modulation of cognitive functions in healthy subjects and patients with brain disorders, ranging from neurological and psychiatric 
diseases to stroke-induced dysfunction (1-4). The stimulation parameters and electrode configurations varied greatly, and the optimal protocol of tDCS remains unclear. The present study is based on the idea that modulation of neuronal spiking due to tDCS-induced membrane potential changes (5-8) could be more effective in modulating neural circuits that are activated at the time of $\operatorname{tDCS}(2,8)$. Using a mouse rotarod learning paradigm, we examined this idea in normal wild-type mice as well as stroke model mice that were produced by MCAO. In both cases, we found that motor learning was markedly enhanced by applying anodal but not cathodal tDCS at the primary motor cortex (M1), and the learning enhancement could not be transferred to a different motor learning task. These results, together with in vivo calcium imaging of cortical neuronal activity in response to tDCS, support the notion that the efficacy of tDCS could be increased if its application is temporally conjunctive with the execution of targeted brain functions.

Mice were subjected to a rotarod running task that began each day with a 5-min familiarization period for rotarod running at a constant low rotation speed, followed by three 5-min trials with gradually increasing speed (4 to $40 \mathrm{rpm}$, day $1 \& 2 ; 8-80 \mathrm{rpm}$, day $3 \& 4$ ) (9) that were interrupted by 5 min inter-trial intervals (ITIs) off the rotarod (Fig. 1A). Mice were subjected to tDCS at designated time with anodal ("+"”) or cathodal (“-”) 
currents, or without current (sham control "S") (Fig. 1B). The mice normally learned to run on the rotarod over the four training days, as shown by increasing duration of staying on the rotarod (Fig. 1C) and increasing terminal rotor speed when the mouse dropped off the rotarod (Fig. 1D). When tDCS was applied to the right primary motor cortex (M1) during the familiarization period and all three task trials each day ("online" stimulation), we found a significant increase in both the time on the rotarod and the terminal speed, beginning on the second day of training (Fig. 1, C and D, and movie S1 and S2). This enhancement of motor learning remained detectable at 14 but not 28 days after training (fig. S1, A and B). The results were further quantified by the rate of learning, as defined by the normalized difference of the terminal speed between the first and last training trials (Fig. 1E). Doubling the anodal tDCS current magnitude to $0.2 \mathrm{~mA}$ caused occasional convulsion in mice, whereas reducing current magnitude to $0.05 \mathrm{~mA}$ resulted in no learning enhancement (Fig. 1E, and fig. S2, A and B). We thus chose $0.1 \mathrm{~mA}$ for the standard anodal tDCS in this study. Furthermore, we found no enhancement of rotarod learning when the same online anodal tDCS was applied to the primary visual cortex (V1, Fig. 1E, and fig. S3, A and B), indicating site specificity for the tDCS effect. The rotarod learning was not affected by the procedure of surgery and electrode installation, as shown by comparing the learning of mice that were not subjected to the procedure (fig. S4, A and B). 
In contrast to the learning enhancement described above, we found that anodal tDCS (at $0.1 \mathrm{~mA}$ ) applied during all 5-min ITIs before or after rotarod running ("offline" stimulation) had no effect on rotarod learning (Fig. 1, F to H, and fig. S5, A and B). Furthermore, no effect was found when anodal tDCS was applied continuously for 20 min before the task onset (Fig. 1H, and fig. S5, C and D), a protocol often used in clinical research (10). In contrast to anodal tDCS, online cathodal tDCS $(0.1 \mathrm{~mA})$ at M1 also had no effect on rotarod learning (Fig. 1E, and fig. S6, A and B). However, when the cathodal current was increased to $0.2 \mathrm{~mA}$, learning was impaired at the $3^{\text {rd }}$ and $4^{\text {th }}$ day of training (Fig. 1E, and fig. S6, C and D). Unlike that found for anodal tDCS, both online and offline cathodal stimulation at $0.2 \mathrm{~mA}$ resulted in similar impairment of learning (Fig. 1, E and H, and fig. S6, C and D). As shown later, this may be attributed to the long-lasting (>5 min) suppression of neuronal firing by cathodal tDCS. Taken together, these findings show that tDCS could bi-directionally modulate rotarod learning, and that the enhancement effect is significant only when anodal tDCS is applied in conjunction with the performance of the rotarod task.

The effect of online anodal tDCS on motor learning may be attributed to specific enhancement of rotarod running skill or improvement of general 
motor coordination. To address this issue, we introduced beam-walking learning task, in which the mouse was given a short familiarization period for walking along a wide beam $(25 \mathrm{~mm})$, followed by 3 trials of walking on a narrow beam ( $7 \mathrm{~mm}$, day $1 \& 2 ; 3 \mathrm{~mm}$, day $3 \& 4$ ) (Fig. $2 \mathrm{~A})$. The learning was shown by the gradual reduction of the mean transverse time and the mean number of hindlimb slips during the beam walking, and the learning rate was quantified by the normalized difference of the mean number of slips between the last and the first beam-walking trial on the 3 $\mathrm{mm}$ beam. In the first set of experiments, we compared beam walking learning before and after 4 days of rotarod learning, and found no effect of rotarod training on learning the beam walking, as shown the reduction of the average total number of hindlimb slips (fig. S7, A to C), implying that the learning was specific to rotarod running. In the second set of experiments, we trained the mice to perform both rotarod running and beam walking ("dual tasks") on each day for 4 days, and found that the extent of learning for the beam walking was not affected by the rotarod learning, and reached a level comparable to that of beam-walking training alone (fig. S8, A to C). Thus, there was no transfer of learning from rotarod running to beam walking. Importantly, when we enhanced the rotarod learning by online anodal tDCS, no elevated learning of beam walking was observed during the dual-task training (Fig. 2, B to E ). Conversely, when the learning of beam walking was enhanced by online anodal tDCS (fig. 
S9, A and B, and movie S3 to S6), no enhanced learning of rotarod running was observed (fig. S10, A to D). Thus, the learning enhancement of online anodal tDCS during rotarod learning was specific to the rotarod task, rather than a general improvement of motor skill. In contrast to this specific anodal tDCS effect, we found that both online and offline cathodal tDCS during rotarod training had suppressive effects on learning both rotarod running (Fig. 2, F and $\mathrm{H}$ ) and beam walking (Fig. 2, G and I).

We next examined the action of tDCS on the activity of M1 neurons using transcranial in vivo two-photon calcium imaging. We used thy-1 transgenic mice expressing the calcium-sensitive fluorescent protein GCaMP6s in cortical neurons, and monitored spiking activity of individual neurons by measuring transient elevation of GCaMP6s fluorescence (11). After the skull-thinning procedure, we monitored the activity of a population of cortical neurons in the superficial layers of M1 in head-fixed mice on a rotating treadmill (at a constant rotation speed of $15 \mathrm{rpm}$ ), with tDCS applied through the thinned skull covered by a saline pool (Fig. 3A). We observed substantial spontaneous activity of M1 neurons (Fig. 3B), as reflected by pulsatile changes of fluorescence signals $\left(\Delta \mathrm{F} / \mathrm{F}_{0}\right)$. Furthermore, anodal tDCS resulted in a gradual increase of firing activity in many neurons during two consecutive 5-min application of anodal tDCS (Fig. 3, C and D, and movie S7). The tDCS-induced increase in spiking 
activity was significantly higher for mice running on the rotating treadmill ("task") than those in a quiet resting state ("rest", Fig. 3, D and E). The lower current magnitude $(25 \mu \mathrm{A})$ was used, based on current density estimates at the cortical surface under the thinned-skull condition. In contrast, cathodal tDCS $(50 \mu \mathrm{A})$ applied to the same mice suppressed the firing of the same group of neurons during both "task" and "rest" periods (Fig. 3, D and F, and movie S8).

The results from all transgenic GCaMP6s mice $(n=6)$ were summarized by plotting the average fluorescence change $\left(\Delta \mathrm{F} / \mathrm{F}_{0}\right)$ during the 2-min baseline period prior to tDCS and the last 2-min of tDCS, for two consecutive trials under the task and rest conditions. Significant elevation and suppression of fluorescence signals were found for anodal and cathodal tDCS during the task period, respectively (Fig. 3, E and F). We noted that changes in the average fluorescence signal subsided gradually after each tDCS offset, and that the suppressive effect of cathodal tDCS persisted for a longer duration than the enhancement effect of anodal tDCS (Fig. 3G). This may account for the offline suppressive effect on the rotarod learning described above using only 5-min ITI in the present paradigm. Taken together, these results support the notion that anodal and cathodal tDCS modulate neuronal firing by inducing depolarization and hyperpolarization of cortical neurons, respectively, consistent with previous findings on 
isolated brain slices (12-14). When applied at the time of specific motor circuit activation, as that occurred during rotarod running, anodal and cathodal tDCS could facilitate and reduce learning-associated modification of specific motor circuits in M1, via enhancing and reducing correlated firing that induces Hebbian long-term potentiation (LTP) of synapses within these circuits, respectively.

There are previous reports on the use of tDCS in alleviating neurologic disorders, including stroke $(4,15)$. Meta-analyses of published reports $(16$, 17) have shown a large variability in the clinical efficacy of tDCS that may be attributed to differences in the tDCS protocol and stroke condition. We thus examined the effect of tDCS on motor learning in a relatively defined mouse model of stroke. A standard middle cerebral artery occlusion (MCAO) in the mouse' left hemisphere was used to induce a large lesion within the left somatosensory cortex and part of the motor cortex at one day after 60 or $90 \mathrm{~min}$ of MCAO (Fig. 4A). When these mice were subjected to rotarod learning 14 days after MCAO (Fig. 4A), we found their motor coordination was significantly impaired, as shown by overall reduction in the time on the rotarod and the rate of rotarod learning, as compared to control mice that underwent MCAO surgery without sustained artery occlusion (Fig. 4, B and C). Furthermore, online anodal tDCS at the left perilesional M1 region (Fig. 4A) largely restored the learning of motor 
coordination and running on the rotarod (Fig. 4, B, C and E, movie S9 and S10). In contrast, offline anodal tDCS and online or offline cathodal tDCS at the same site had no effect on learning motor coordination and rotarod running in this stroke model (fig. S11, A and B, and S12, A and B). Furthermore, online cathodal tDCS at the opposite site (contralateral to the infarct area) also had no effect on rotarod learning in stroke model mice (fig. S12, C and D).

In the absence of tDCS, 90-min MCAO impaired motor learning of both rotarod running and beam walking, as compared to control mice (Fig. 4, B to F). However, the mice that showed rotarod learning restoration by online anodal tDCS did not exhibit any improvement in learning beam walking, as compared to those subjected to sham tDCS treatment during rotarod running (Fig. 4, B to F). In contrast, offline anodal tDCS during rotarod training had no effect on learning both rotarod running and beam walking (Fig. 4, G and H, and fig. S13, A to D). Therefore, the restoration of rotarod learning in MCAO mice by anodal tDCS was task-specific, rather than a general restoration of learning for motor skills. Based on the above finding of elevated neuronal firing induced by anodal tDCS, the restoration of rotarod learning may involve specific enhancement of residual neural circuits after $\mathrm{MCAO}$ that were activated during rotarod running, without affecting those underlying beam walking. 
The timing of tDCS relative to the targeted task performance has been addressed in previous studies of healthy human subjects and stroke patients, but conflicting results have been reported, as summarized by various metaanalyses $(16,17)$. For examples, online but not offline anodal tDCS of M1 during motor sequence task was found to enhance motor learning, while online cathodal tDCS had no or opposite effects $(18,19)$. However, another study using offline anodal tDCS prior to the motor task showed enhancement effect on motor learning (20). In cases of prolonged tDCS, the effects on human motor cortex could last for hours (21) and even days (22), and the timing of tDCS becomes less irrelevant. Our present results underscore the importance of online application of anodal tDCS during task performance when brief episodes of tDCS was used.

Previous studies on healthy human subjects have shown that anodal tDCS enhanced cognition or motor learning (23-26) and these effects were specific to different level of task difficulty $(27,28)$ or the site $(26,29)$ of tDCS. We found that tDCS on M1 specifically enhanced learning of rotarod task, without affecting learning of beam walking. Thus, even within the motor domain, conjunctive tDCS could exert modulation of specific motor functions. Taken together, our results strongly support the notion that functional specificity of tDCS depends on not only the spatiotemporal 
pattern of electric fields applied (30) but also on the temporal conjunction of tDCS with the activation of specific circuits underlying the targeted function.

\section{ACKNOWLEDGMENTS}

We thank Drs. Yang Dan, Chun Xu, Liping Wang, Huatai Xu for suggestions; Drs. Huanhuan Zeng for technical support; Dr. Zhijie Wang for providing the head-fixation holder and plates; and Dr. Ninglong Xu for providing GCaMP6s transgenic mice. Funding: this work was supported by International Partnership Program of Chinese Academy of Sciences (153D31KYSB20170059), the Strategic Priority Research Program of the Chinese Academy of Sciences (XDB32070100), Key Research Program of Frontier Sciences, Chinese Academy of Sciences (QYZDY-SSWSMCO01), Shanghai Municipal Science and Technology Major Project (2018SHZDZX05), the Shanghai Key Basic Research Project (18JC1410100), and the Shanghai Key Basic Research Project (16JC1420201). Author Contributions: Y.W., M.P, and Y.W. designed the study, Y.W., J.W. and L.W. performed the experiments; Q.Z., K.X., Y.W. and Y.W. wrote the data processing program and provided technical help; Q.X. assisted with the design of experiment; Q.Y. helped managing the mouse colony; and Y.W, M.P., and Y.W. wrote the manuscript.

Competing interests: The authors declare no competing interests. 


\section{SUPPLEMENATARY MATERIALS}

\section{Materials and Methods}

Figs. S1 to S13

Movies S1 to S10

References (31-39) 


\section{Figure Legends}

A

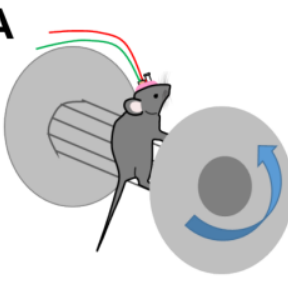

$$
\text { C }
$$

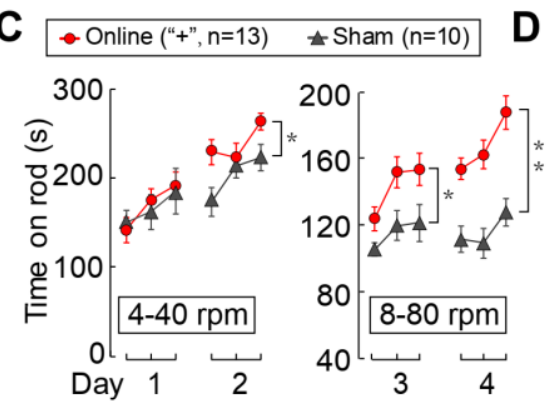

$\mathbf{F} \rightarrow$ Offline $("+", n=9) \quad$ Sham $(\mathrm{n}=11)$ G

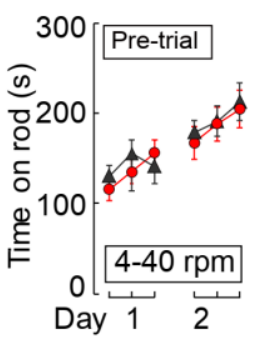

D
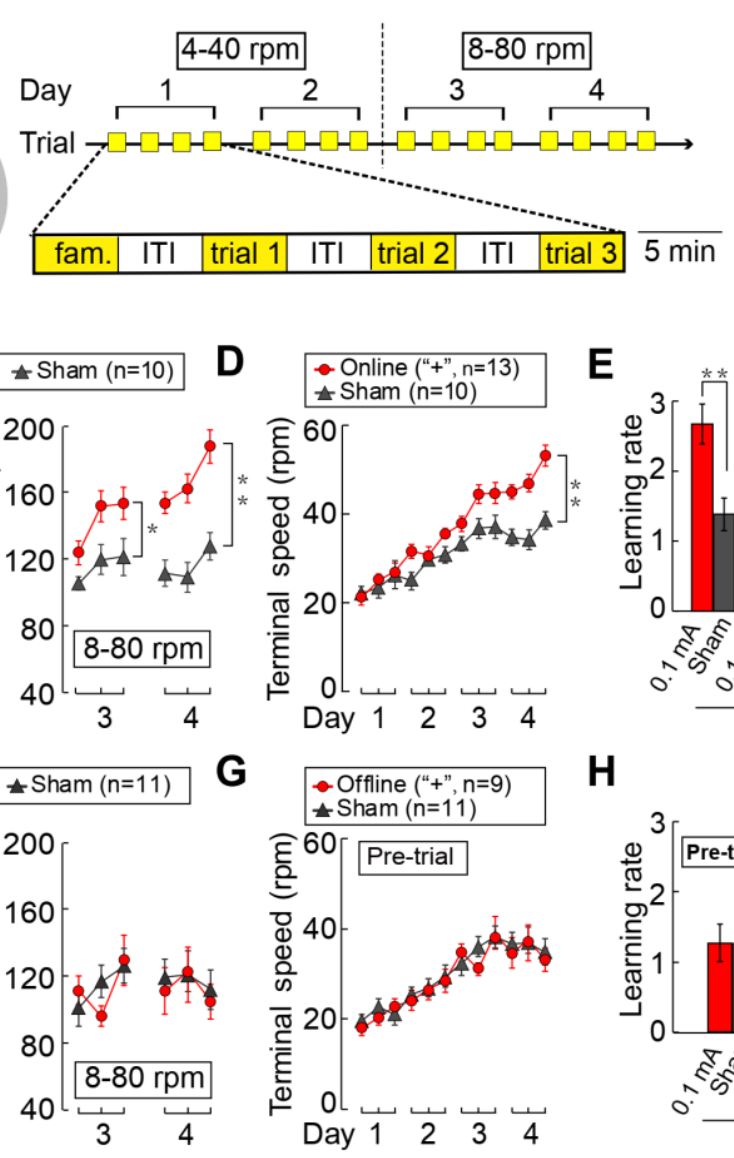

B

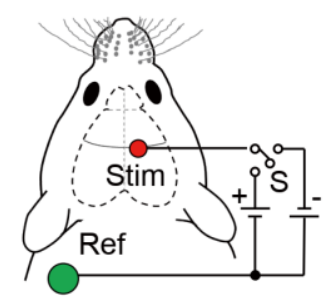

Online

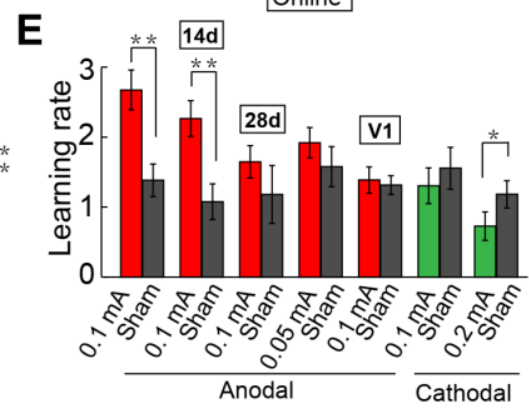

H

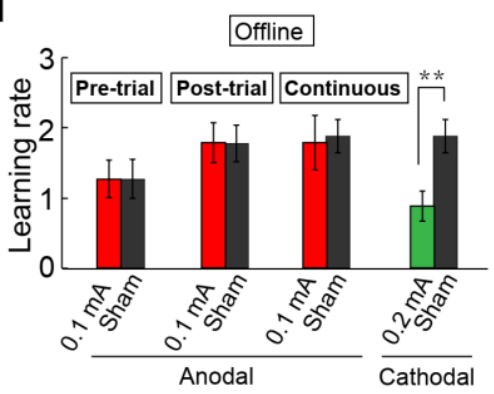

Fig. 1. Effects of tDCS on mouse learning of the rotarod running task.

(A) Training Protocol: The mouse was subjected each day to a 5-min familiarization trial at a constant low speed, followed by three 5-min trials (separated by 5 min inter-trial interval) at linearly increasing rotation speed. (day 1 \& 2: 4 - $40 \mathrm{rpm}$; day $3 \&$ 4: 8-80 rpm). (B) Schematic diagram depicting the electrode configuration. "Stim": tDCS electrode. "Ref": reference electrode. "S": sham (no current). “+": anodal. "-": cathodal. (C) The average time of staying on the rotarod during each trial. (D) The 
terminal rotation speed at which mice fell off the rotarod during each trial. “Online": anodal tDCS (0.1 mA) was applied during each trial. "n": total number of mice examined. (E) Summary of results showing the learning rate, as defined by the normalized difference of terminal speed between the last and the first trial of the entire training period. Data depict standard 4day training with (color bars) and without (sham, black bars) online anodal (or cathodal) tDCS, applied to M1 at different current amplitudes. "14d" and "28d" refer to results obtained with 3 additional training trials at 14 and 28 days after training. "V1": the tDCS was applied to V1 instead of M1. (F to $\mathbf{H})$ The results from experiments in which tDCS was applied during ITIs, presented in the same manner as those described above for CE. "Pre-trial" and "Post-trial" refer to the average values obtained with tDCS applied during ITIs before and after each trial, respectively. "Continuous", 20-min continuous tDCS applied before the familiarization trial. Error bars, SEM. Significant difference was found between the data sets connected by lines ( “*”, p<0.05; “**” , p<0.01; C, D, F, G: twoway ANOVA; E, H: unpaired $t$ test). 
A

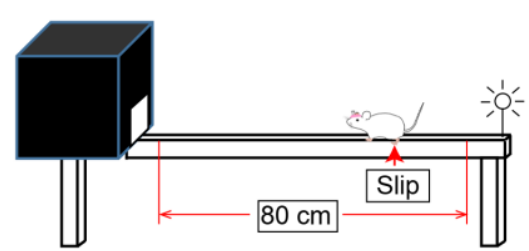

B
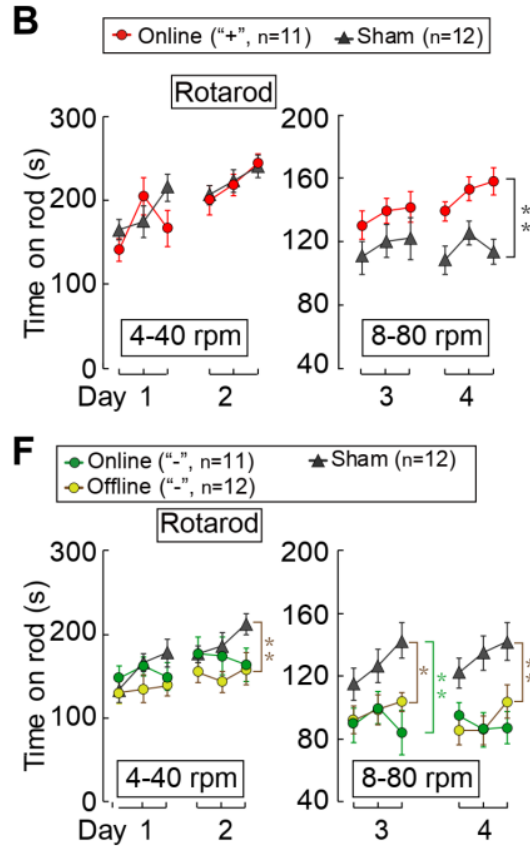

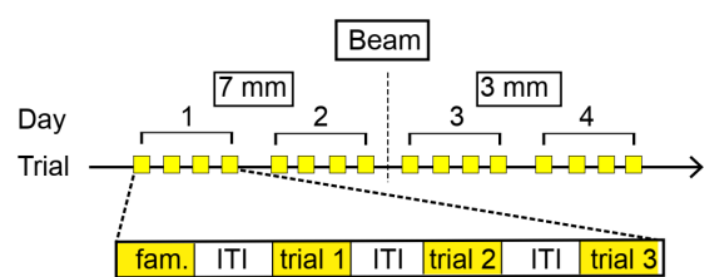

C

- Online ("+", $n=11) \nRightarrow$ Sham $(\mathrm{n}=12)$
(during rotarod)

D Rotarod

E Beam
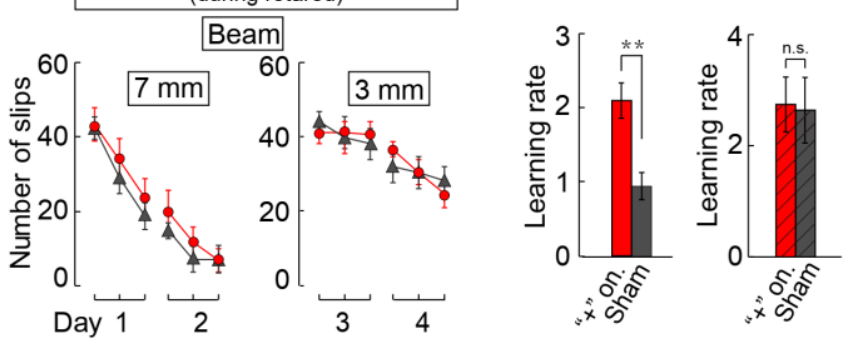

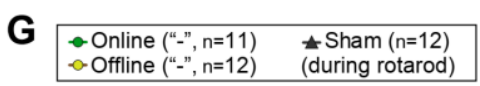

Beam

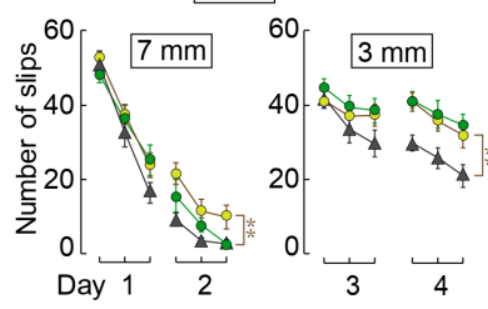

H Rotarod I Beam
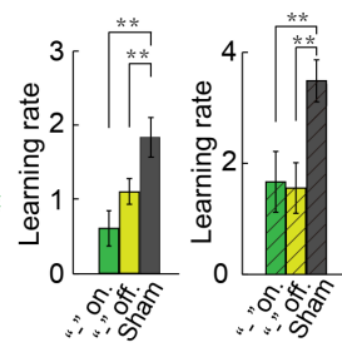

Fig. 2. Effects of tDCS-induced modulation of rotarod learning on the

learning of beam walking. (A) Experimental protocol of beam walking.

The mice were subjected to anodal online tDCS in the same manner as that described in Fig. 1C, except that rotarod task was followed by a beam walking learning task in the absence of tDCS. The mice were subjected to wide beam $(25 \mathrm{~mm})$ familiarization, followed by three trials on the thinner beam (day 1 \& day 2, $7 \mathrm{~mm}$; day $3 \&$ day $4,3 \mathrm{~mm}$ ). (B and $\mathbf{C}$ ) Data from dual-task experiments. Average time on the rotarod (in B) was presented as that in Fig.1C. The reduction in the average frequency of hindlimb slips (in C) during the 4-day training of beam walking. Note that online anodal tDCS during rotarod running improved rotarod learning (in B), but had no 
effect on learning beam walking (in C). (D and E) Summary of results showing rotarod learning rate (see Fig.1E) and beam walking learning rate, as defined by normalized difference of the slip frequencies between the last and the first trial of the beam walking on the 3-mm beam. (F to I) Results of rotarod learning and beam walking learning by cathodal online (or offline) tDCS during rotarod learning. Error bars, SEM. Significant difference was found between the data sets connected by lines ( “*”, p< $0.05 ; “ * * ”, \mathrm{p}<0.01 ; \mathrm{B}, \mathrm{C}, \mathrm{E}, \mathrm{F}$ : two-way ANOVA; D, G: unpaired $t$ test). 

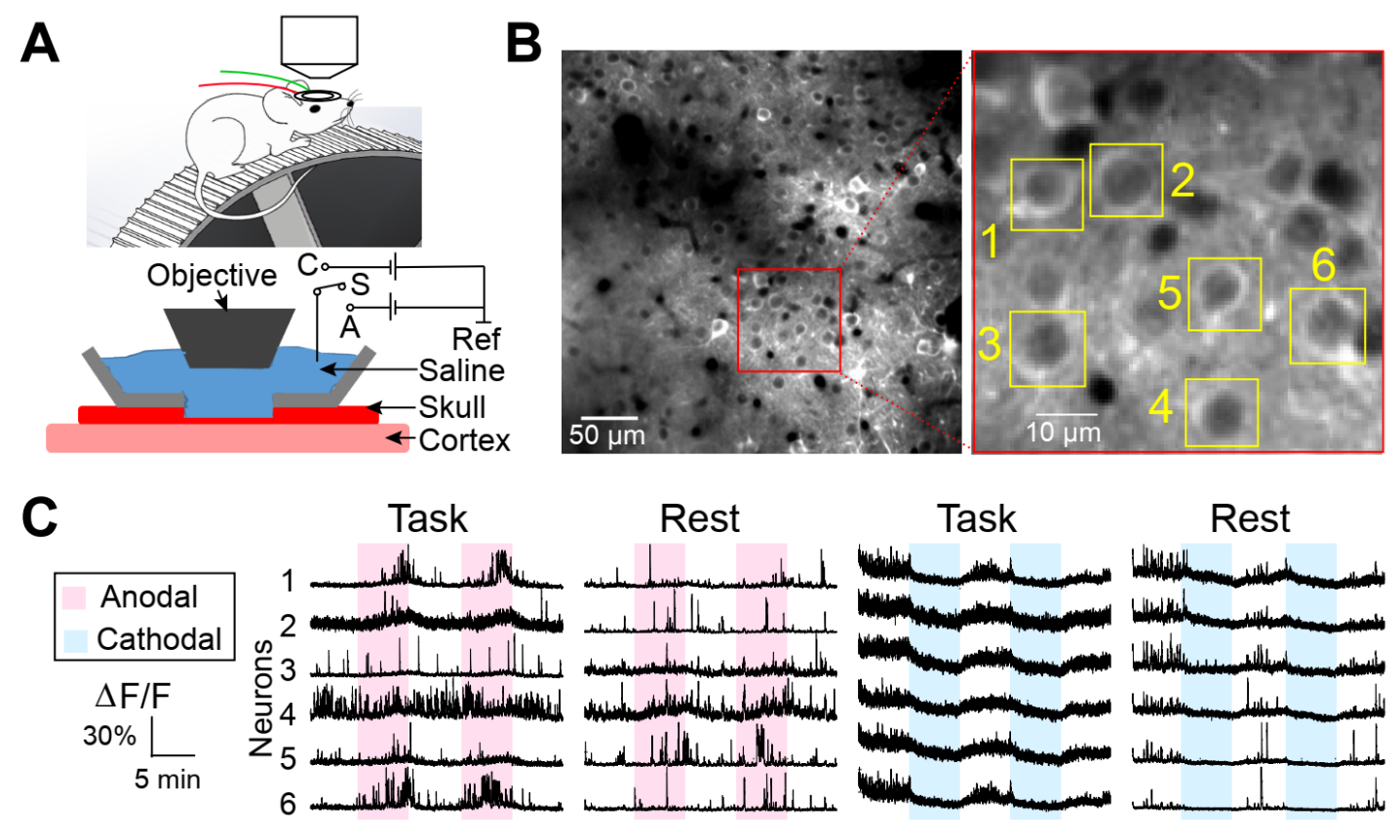

D
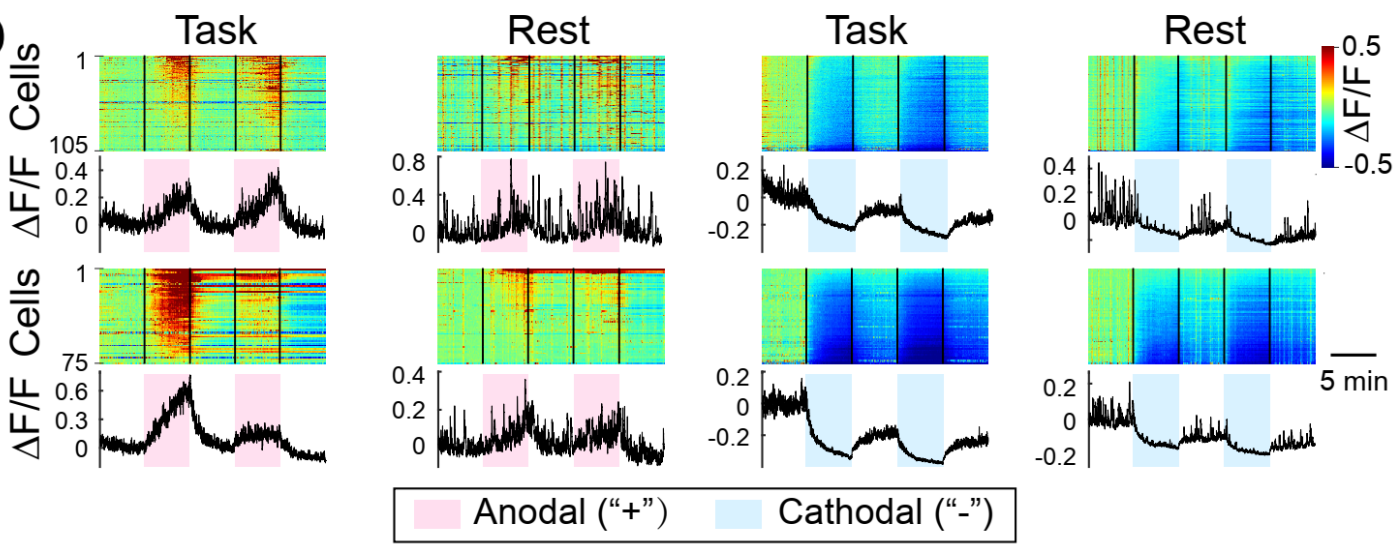

Anodal ("+")

Cathodal $(-)$
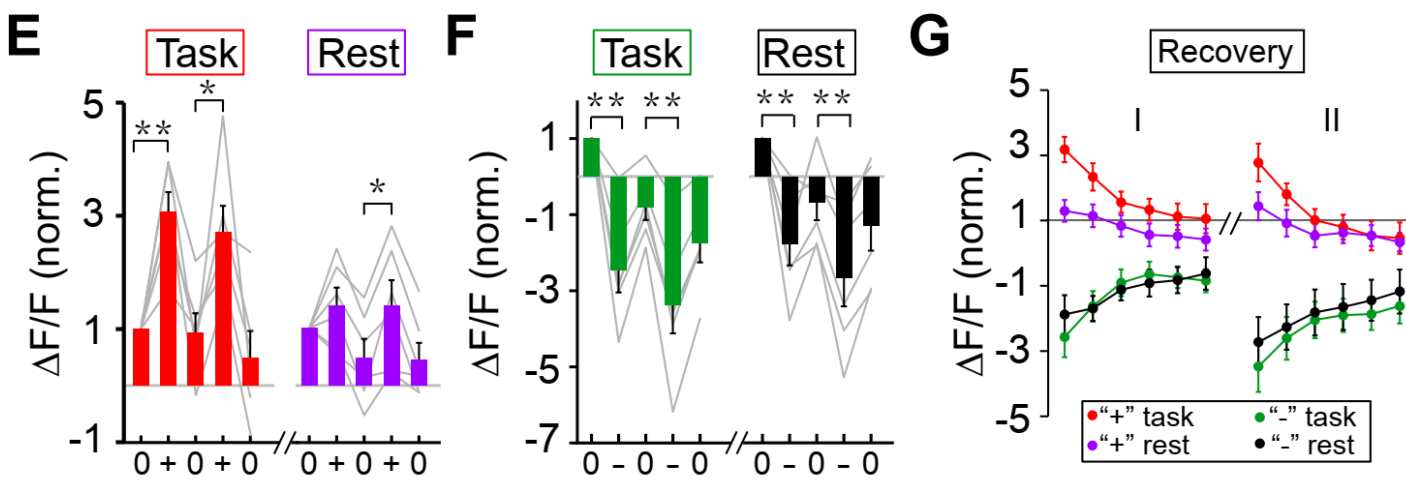

Fig. 3. Transcranial two-photon imaging of tDCS-induced modulation

of cortical neuronal activity. (A) Schematic diagram depicting the optical window over the thinned skull, for two-photon imaging of M1 neurons in a head-fixed mouse on the rotating treadmill. (B) Example 
images of Thy1-GCaMP6s-expressing neurons in M1, viewed through the optical window. Scale bar: $50 \mu \mathrm{m}$. Image on the right: higher resolution image of the red-boxed region on the left, showing fluorescence of individual layer 2/3 neurons. Scale bar: $10 \mu \mathrm{m}$. (C) Changes of GCaMP6s fluorescence $\left(\Delta \mathrm{F} / \mathrm{F}_{0}\right)$ with time monitored at six M1 neurons (marked by yellow squares in B). Pink area: time of anodal tDCS (at $25 \mu \mathrm{A}$ ); blue area: time of cathodal tDCS (at $50 \mu \mathrm{A}$ ); "Task": walking on steady rotating treadmill; "Rest": mouse in a stereotaxic frame. (D) Fluorescence changes of all fluorescently labelled cells within the imaged field, recorded from two example mice. The amplitude of $\Delta \mathrm{F} / \mathrm{F}_{0}$ for each cell with time was color-coded with the scale shown on the right. The cells were ordered according to the peak values of $\Delta \mathrm{F} / \mathrm{F}_{0}$. Traces below represent average $\Delta \mathrm{F}$ $/ \mathrm{F}_{0}$ for all cells shown above. The same group of cells were monitored before and after two episodes of anodal and cathodal tDCS sequentially, under the task and rest conditions. (E) Summary on tDCS-induced GCaMP6s fluorescence changes for data from all mice $(n=6)$. Average fluorescence changes $\left(\Delta \mathrm{F} / \mathrm{F}_{0}\right)$ during the last 2-min of tDCS were normalized by the average values during the $2-\mathrm{min}$ baseline period prior to tDCS, for two consecutive trials under the task and rest conditions. Data for the same set of neurons in each mouse were connected by lines. Significant difference was marked (“*”, $\mathrm{p}<0.05$; “**”, $\mathrm{p}<0.01$; paired $t$ test). (F) Post-treatment persistence of tDCS effects was shown by the 
average fluorescence changes with time, normalized by the values at the time of termination of anodal or cathodal tDCS, for task and rest conditions $(n=6$ mice). Note the slower decay of the inhibitory effect after cathodal tDCS.

A
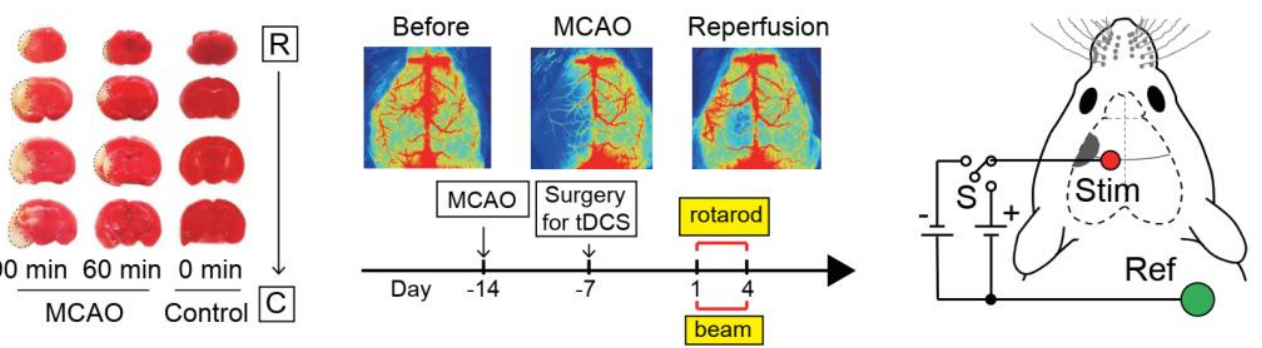

B
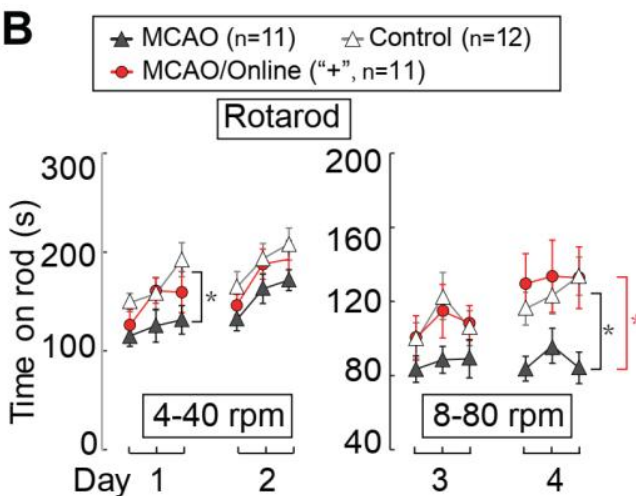

C
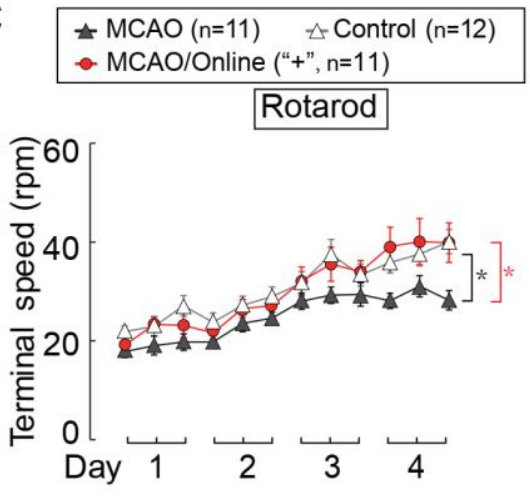

D
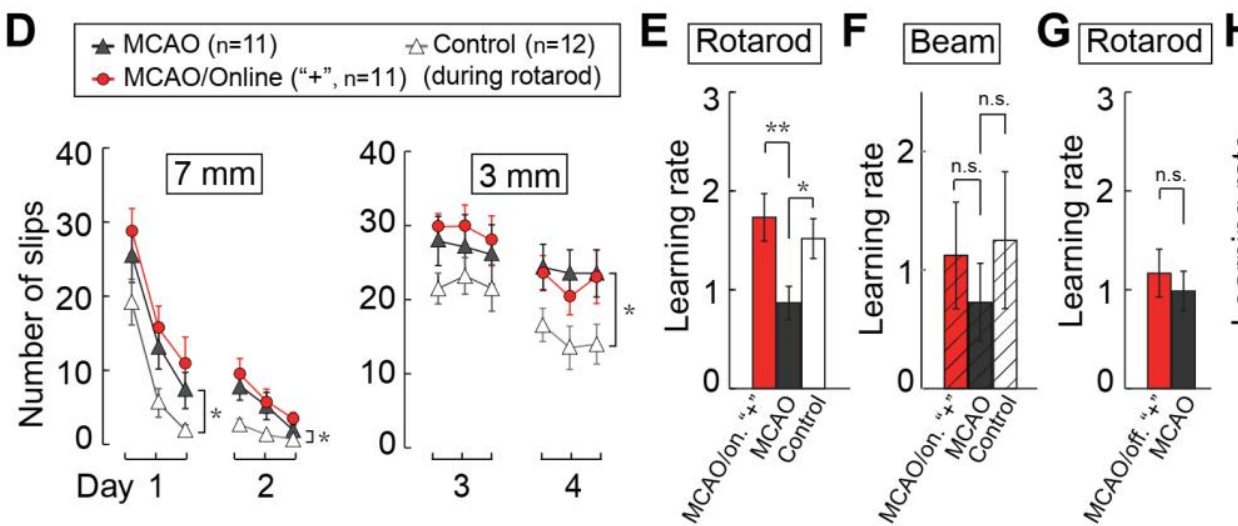

\section{Heam}

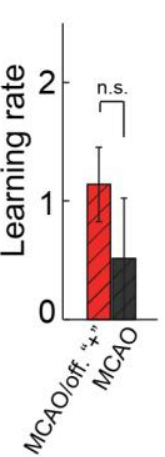

Fig. 4. Task-specific restoration of rotarod learning by online anodal 
tDCS in MCAO mice. (A) TTC staining (left) and laser speckle contrast imaging (LSCI) (right) showing the lesion induced by MCAO, which was performed for 90,60 or 0 min prior to reperfusion. The time schedule of MCAO, surgery for tDCS, and training for rotarod running and beam walking is shown. Schematic diagram on the right depicts the placement of tDCS electrodes in MCAO mice. Infarct area marked in gray, and the stimulation electrode ("Stim") covered parts of M1 and somatosensory cortex. (B and $\mathbf{C})$ The average time on and terminal speed of the rotarod, for MCAO mice treated with online anodal tDCS, sham-stimulation, and Sham-MCAO surgery (control) in dual-task experiments, in which tDCS was applied only during rotarod running. Data presented in the same manner as in Fig.1, C and D. "MCAO": mice subjected to 90-min occlusion in MCA; "Control" (Sham-MCAO): mice subjected to the same surgery with no occlusion in MCA; "MCAO/online": MCAO mice subjected online tDCS during rotarod running. (D) The average frequency of hindlimb slips (contralateral to the lesion) during beam walking. (E and F) Learning rate for rotarod running and beam walking in MCAO mice subjected to online anodal tDCS. $(\mathbf{G}$ and $\mathbf{H})$ Learning rate of rotarod and beam walking in MCAO mice subjected to offline anodal tDCS. Error bars, SEM. Significant difference was found between the data sets connected by lines ( “*”, p<0.05; “**” , p<0.01; C, D, E: two-way ANOVA; F, G, H, I: unpaired $t$ test). 


\section{References and Notes}

1. S. Bestmann, V. Walsh, Transcranial electrical stimulation. Curr Biol 27, R1258-R1262 (2017).

2. R. Polania, M. A. Nitsche, C. C. Ruff, Studying and modifying brain function with non-invasive brain stimulation. Nat Neurosci 21, 174-187 (2018).

3. M. F. Kuo, W. Paulus, M. A. Nitsche, Therapeutic effects of non-invasive brain stimulation with direct currents (tDCS) in neuropsychiatric diseases. Neuroimage 85 Pt 3, 948-960 (2014).

4. A. Gomez Palacio Schjetnan, J. Faraji, G. A. Metz, M. Tatsuno, A. Luczak, Transcranial direct current stimulation in stroke rehabilitation: a review of recent advancements. Stroke Res Treat 2013, 170256 (2013).

5. M A Nitsche, W. Paulus, Excitability changes induced in the human motor cortex by weak transcranial direct current stimulation. J Physiol 527 Pt 3, 633-639 (2000).

6. F. Bolzoni, M. Baczyk, E. Jankowska, Subcortical effects of transcranial direct current stimulation in the rat. J Physiol 591, 4027-4042 (2013).

7. A. Rahman, B. Lafon, M. Bikson, Multilevel computational models for predicting the cellular effects of noninvasive brain stimulation. Prog Brain Res 222, 25-40 (2015).

8. M. Bikson, A. Name, A. Rahman, Origins of specificity during tDCS: anatomical, activityselective, and input-bias mechanisms. Front Hum Neurosci 7, 688 (2013).

9. P. E. Rothwell et al., Autism-associated neuroligin-3 mutations commonly impair striatal circuits to boost repetitive behaviors. Cell 158, 198-212 (2014).

10. D. Bennabi et al., Transcranial direct current stimulation for memory enhancement: from clinical research to animal models. Front Syst Neurosci 8, 159 (2014).

11. T. W. Chen et al., Ultrasensitive fluorescent proteins for imaging neuronal activity. Nature 499, 295-300 (2013).

12. L. F. Medeiros et al., Neurobiological effects of transcranial direct current stimulation: a review. Front Psychiatry 3, 110 (2012).

13. B. Fritsch et al., Direct current stimulation promotes BDNF-dependent synaptic plasticity: potential implications for motor learning. Neuron 66, 198-204 (2010).

14. G. Kronberg, M. Bridi, T. Abel, M. Bikson, L. C. Parra, Direct Current Stimulation Modulates LTP and LTD: Activity Dependence and Dendritic Effects. Brain Stimul 10, 51-58 (2017).

15. Claire Allman et al., Ipsilesional anodal tDCS enhances the functional benefits of rehabilitation in patients after stroke. Sci Transl Med 8, 330re1 (2016).

16. Felipe Fregni et al., Evidence-based guidelines and secondary meta-analysis for the use of transcranial direct current stimulation (tDCS) in neurological and psychiatric disorders. Int $J$ Neuropsychopharmacol 2020, pyaa051 (2020).

17. Xi Bai et al., Different Therapeutic Effects of Transcranial Direct Current Stimulation on Upper and Lower Limb Recovery of Stroke Patients with Motor Dysfunction: A Meta-Analysis. Neural Plast 2019, 1372138 (2019).

18. Michael A Nitsche et al., Facilitation of implicit motor learning by weak transcranial direct current stimulation of the primary motor cortex in the human. J Cogn Neurosci 15, 619-626 (2003).

19. C. J. Stagg et al., Polarity and timing-dependent effects of transcranial direct current stimulation 
in explicit motor learning. Neuropsychologia 49, 800-804 (2011).

20. D. J. Kidgell, A. M. Goodwill, A. K. Frazer, R. M. Daly, Induction of cortical plasticity and improved motor performance following unilateral and bilateral transcranial direct current stimulation of the primary motor cortex. Bmc Neurosci 14, 64 (2013).

21. M. A. Nitsche, W. Paulus, Sustained excitability elevations induced by transcranial DC motor cortex stimulation in humans. Neurology 57, 1899-1901 (2001).

22. Janine Reis et al., Noninvasive cortical stimulation enhances motor skill acquisition over multiple days through an effect on consolidation. Proc Natl Acad Sci U S A 106, 1590-1595 (2009).

23. S. C. Andrews, K. E. Hoy, P. G. Enticott, Z. J. Daskalakis, P. B. Fitzgerald, Improving working memory: the effect of combining cognitive activity and anodal transcranial direct current stimulation to the left dorsolateral prefrontal cortex. Brain Stimul 4, 84-89 (2011).

24. Suk Hoon Ohn et al., Time-dependent effect of transcranial direct current stimulation on the enhancement of working memory. Neuroreport 19, 43-47 (2008).

25. J. Gill, P. P. Shah-Basak, R. Hamilton, It's the thought that counts: examining the task-dependent effects of transcranial direct current stimulation on executive function. Brain Stimul 8, 253-259 (2015).

26. C. M. Saucedo Marquez, X. Zhang, S. P. Swinnen, R. Meesen, N. Wenderoth, Task-Specific Effect of Transcranial Direct Current Stimulation on Motor Learning. Frontiers in Human Neuroscience 7, 333 (2013).

27. P. A. Pope, J. W. Brenton, R. C. Miall, Task-Specific Facilitation of Cognition by Anodal Transcranial Direct Current Stimulation of the Prefrontal Cortex. Cerebral Cortex 25, 45514558 (2015).

28. P. A. Pope, R. C. Miall, Task-specific facilitation of cognition by cathodal transcranial direct current stimulation of the cerebellum. Brain Stimulation 5, 84-94 (2012).

29. S. Karok, D. Fletcher, A. G. Witney, Task-specificity of unilateral anodal and dual-M1 tDCS effects on motor learning. Neuropsychologia 94, 84-95 (2017).

30. Alicia Nunez Vorobiova, Ivan Pozdniakov, M. Feurra, Transcranial Direct Current Stimulation Effects on Memory Consolidation: Timing Matters. eNeuro 6, ENEURO.0481-0418 (2019). 